\title{
SPECTRAL RADIUS OF AN ABSOLUTELY CONTINUOUS OPERATOR
}

\author{
ARNOLD LEBOW
}

Abstract. An operator $T$ on a Hilbert space is said to be absolutely continuous if, for every pair of vectors $(x, y)$ and every nonnegative integer $n,\left\langle T^{n} x, y\right\rangle$ is the $n$th Fourier coefficient of an $L_{1}(0,2 \pi)$ function $F_{r y}$ :

$$
\left\langle T^{n} x, y\right\rangle=\frac{1}{2 \pi} \int_{0}^{2 \pi} F_{2 y}(\theta) e^{-i n \theta} d \theta .
$$

The main result of this paper is that if $F_{x y}$ is in $\bigcup\left\{L_{p}: p>1\right\}$ for all $x$ and $y$ then $T$ has spectral radius less than one.

The operators considered here form a special case of the class of polynomially bounded operators. An operator $T$ is polynomially bounded if

$$
\|\mathscr{P}(T)\| \leqq K \sup \left\{\left|\mathscr{P}\left(e^{i \theta}\right)\right|\right\}
$$

for every polynomial $\mathscr{P}$, where $K$ is a constant independent of $\mathscr{P}$. It has been observed [2] that this condition is equivalent to the existence of complex measures $\mu_{x y}$ such that

$$
\left\langle T^{n} x, y\right\rangle=\int_{0}^{2 \pi} e^{-i n \theta} d \mu_{x y}(\theta)
$$

These measures provide a natural method of classifying special types of polynomially bounded operators. For example, call the operator $T$ of "positive type" if $\mu_{x x}$ can be chosen to be a positive measure. It is not difficult to show that this implies that $T$ is a contraction.

At this time it should be pointed out that the measure $\mu_{x y}$ is not unique; indeed, we know only half its Fourier coefficients. However, it follows from the F. and M. Riesz Theorem that the singular part of $\mu_{x y}$ is unique. Call a polynomially bounded operator absolutely continuous if, for every $x$ and $y$, the singular part of $\mu_{x y}$ vanishes. It would appear that this concept could be refined further by consideration of absolutely continuous operators such that the Radon-Nikodym derivative of $\mu_{x y}$ is in $L_{p}$. We

Received by the editors December 7, 1971 and, in revised form, April 28, 1972.

AMS 1970 subject classifications. Primary 47A25, 47A30; Secondary 46J15, 46L15.

Key words and phrases. Polynomially bounded, absolutely continuous, RadonNikodym derivative, $H^{p}$-space, spectral radius.

(C) American Mathematical Society 1973 
shall show that there is in fact no distinction between these classes when $p>1$.

LEMMA 1. Let $T$ be an operator, and $p \geqq 1$. If $F_{x y}$ is a function in the Hardy space $H_{p}$ such that

$$
\left\langle T^{n} x, y\right\rangle=\frac{1}{2 \pi} \int_{0}^{2 \pi} F_{x y}(\theta) e^{-i n \theta} d \theta \quad(n=0,1,2, \cdots)
$$

for all $x$ and $y$; then there is a constant $C$ such that

$$
\left\|F_{x y}\right\|_{p} \leqq C\left(\|x\|^{2}+\|y\|^{2}\right)^{1 / 2} .
$$

Proof. The map $F$ from $\mathrm{H} \oplus H^{*}$ to $H_{p}$ defined by $F(x, y)=F_{x y}$ is linear and defined on all of a Banach space so it is sufficient to show that $F$ has a closed graph in order to conclude that $F$ is bounded, which is what the lemma requires. Thus if we assume that $x_{k} \rightarrow x, y_{k} \rightarrow y$, in Hilbert space, and $F\left(x_{k}, y_{k}\right) \rightarrow f$ in $H_{p}$ we need to show that $f=F_{x y}$. Since both functions $f$ and $F_{x v}$ are in $H_{p}$ it is enough to show that their positive Fourier coefficients are equal:

$$
\begin{aligned}
\frac{1}{2 \pi} \int e^{-i n \theta} F_{x y}(\theta) d \theta & =\left\langle T^{n} x, y\right\rangle=\lim _{k}\left\langle T^{n} x_{k}, y_{k}\right\rangle \\
& =\lim \frac{1}{2 \pi} \int e^{-i n \theta} F\left(x_{k}, y_{k}\right)(\theta) d \theta=\frac{1}{2 \pi} \int e^{-i n \theta} f(\theta) d \theta
\end{aligned}
$$

LEMMA 2. Let $X$ be a Banach space and $A$ a bounded linear transformation of $X$ into $L_{1}$. If $A(X)$ is contained in $\bigcup\left\{L_{p}: p>1\right\}$, then $A(X)$ is contained in some $L_{p}, p>1$.

Proof. The referee has pointed out that this result is a corollary of a theorem of Grothendieck [1, p. 225].

THEOREM. If $T$ is an absolutely continuous operator such that $F_{x y}$ is in $\bigcup\left\{L_{p}: p>1\right\}$ for all $x$ and $y$, then $T$ has spectral radius less than one.

Proof. Since the natural projections of $L_{p}$ into $H_{p}$ are continuous for $p>1$ we may assume that each $F_{x y}$ is in $H_{p}$ for some $p>1$ and hence all $F_{x y}$ are in $H_{1}$. By Lemma 1 the mapping $(x, y) \rightarrow F_{x y}$ is continuous and by Lemma 2 there exists a fixed $p>1$ such that $F_{x y}$ is in $H_{p}$. It now follows from Lemma 1 that

$$
\left\|F_{x y}\right\|_{p} \leqq C\left(\|x\|^{2}+\|y\|^{2}\right)^{1 / 2}
$$


Let $\mathscr{P}$ be a polynomial and $x$ and $y$ be unit vectors; then

$$
\langle\mathscr{P}(T) x, y\rangle=\frac{1}{2 \pi} \int_{0}^{2 \pi} \mathscr{P}\left(e^{-i \theta}\right) F_{x y}(\theta) d \theta .
$$

By Hölder's inequality and (1),

$$
|\langle\mathscr{P}(T) x, y\rangle| \leqq \frac{1}{2 \pi}\|\mathscr{P}\|_{q} \cdot\left\|F_{x y}\right\|_{p} \leqq K\|\mathscr{P}\|_{q} .
$$

But this implies that $\|\mathscr{P}(T)\| \leqq K\|\mathscr{P}\|_{q}$. By the spectral mapping theorem and the fact that the uniform norm dominates the spectral radius it is found that

$$
\sup \{|\mathscr{P}(\hat{\lambda})|: \hat{\lambda} \in \sigma(T)\} \leqq K\|\mathscr{P}\|_{Q} .
$$

Since the iterates of $T$ are uniformly bounded we know that $\sigma(T)$ is contained in the closed unit disk. But (2) being true for every polynomial can occur only if $\sigma(T)$ is contained in the open unit disk. Indeed if $e^{i \ell}$ is in $\sigma(T)$ let $\mathscr{P}_{n}(z)=\left[\frac{1}{2}\left(1+e^{-i t} z\right)\right]^{n}$, then $\sup \left\{\mathscr{P}_{n}(\lambda): \lambda \in \sigma(T)\right\}=1$ and $\left\|\mathscr{P}_{n}\right\|_{q} \rightarrow 0$ for $q<\infty$.

This theorem is an extension of a result of M. Schreiber [4] for contractions where the functions $F_{x y}$ are shown to exist by means of dilation theory. In addition Schreiber essentially takes the conclusions of Lemmas 1 and 2 as hypotheses. The converse of the theorem is also true, as may be seen by using a modification of Schreiber's proof of the converse. In fact this shows that the $F_{x y}$ may be chosen to be bounded. An alternate proof of the converse can be obtained from Schreiber's result for contractions and Rota's theorem [3] that operators of spectral radius less than one are similar to contractions.

It is clear that the properties that have been considered here, such as polynomially boundedness, absolute continuity, etc., are similarity invariants. It is still an open question if every polynomially bounded operator is similar to a contraction.

COROLlaRY 1. If $T$ is absolutely continuous and $F_{x y}$ is in $\bigcup\left\{L_{p}: p>1\right\}$ then $T$ is similar to a contraction (of spectral radius less than one).

COROLlaRY 2. The following are equivalent:

(1) $\sum_{0}^{\infty}\left|\left\langle T^{n} x, y\right\rangle\right|^{2}<\infty$ for all $x$ and $y$;

(2) $\sum_{0}^{\infty}\left\|T^{n} x\right\|^{2}<\infty$ for all $x$;

(3) $\left\|T^{n}\right\|^{1 / n} \rightarrow r<1$.

Proof. That (1) implies (3) follows from the theorem by letting $F_{x y}(\theta)=\Sigma\left\langle T^{n} x, y\right\rangle e^{i n \theta}$. The other implications are trivial. 


\section{BIBLIOGRAPHY}

1. G. Köthe, Topologische linear Räume. I, Die Grundlehren der math. Wissenschaften, Band 107, Springer-Verlag, Berlin, 1960; English transl., Springer-Verlag, 1969. MR 24 \#A411; MR 40 \#1750.

2. A. Lebow, A power-bounded operator that is not polynomially bounded, Michigan Math. J. 15 (1968), 397-399. MR 38 \#5047.

3. G.-C. Rota, On models for linear operators, Comm. Pure Appl. Math. 13 (1960), 469-472. MR 28 \#2898.

4. M. Schreiber, Absolutely continuous operators, Duke Math. J. 29 (1962), 175-190. MR 26 \#5424.

Department of Mathematics, Belfer Graduate School, Yeshiva University, NEW YoRK, NEW YORK 10033 